\title{
Nutrición de yodo en escolares de cuatro zonas de Chile en el año 2001
}

\author{
Santiago Muzzo B, Iván Ramírez M, Fresia Carvajal La, \\ Edith Biolley $\mathrm{H}^{\mathrm{a}}$, Laura Leiva $\mathrm{B}^{\mathrm{b}}$.
}

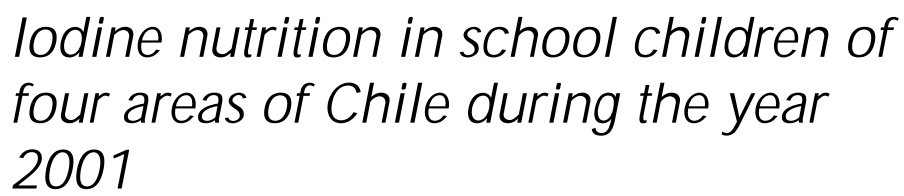

Background: In Chile, the recommendation for salt iodination was reduced from 100 to $40 \mathrm{ppm}$, after detecting high urinary iodine excretion in school age children. Aim: To assess iodine nutrition in school age children after the modification of salt iodination. Subjects and methods: Iodine nutrition was studied in 3712 school age Chilean children of primary level in 4 areas (Calama, Santiago, Temuco and Punta Arenas) through palpation of thyroid gland, urinary iodine excretion and iodine concentration in salt for human consumption of the surveyed areas. Results: A 6.4\% goiter prevalence was found (5,9\% in boys and $6,8 \%$ in girls). Grade Ia goiter was more common (5.0\%), without detecting differences between areas (7.3\% in Calama, 5.9\% in Santiago, 5.9 in Temuco and 6.7 in Punta Arenas). Iodine concentration in salt for human consumption of the areas was adequate according to the recent modification of Chilean legislation $(25.4 \mathrm{mgI} / \mathrm{g}$ salt in Calama, 36.2 in Santiago, 27.7 in Temuco and 33.4 in Punta Arenas). Urinary iodine excretion was very high according to recommendation in boys and girls (2838 and $2852 \mathrm{mgI} / \mathrm{g}$ salt in Calama, 700 and 837 in Santiago, 1318 and 1438 in Temuco, 407 and 312 in Punta Arenas). Conclusions: Endemic goiter is not longer a problem in Chile. Salt iodination is in agreement with present legislation. However, urinary iodine excretion increased in Calama and Temuco, did not change in Santiago and decreased only in Punta Arenas in spite of the changes in the legislation. It is necessary to investigate the causes of the high urinary iodine excretions detected in school age children, and it is very important to have a continuous surveillance of iodine nutrition in Chile (Rev Méd Chile 2003; 131: 1390-8).

(Key Words: Endemic, Goiter; Iodine)



Recibido el 29 de enero, 2003. Aceptado en versión corregida el 23 de septiembre, 2003. Trabajo financiado por Super Sal Lobos (la empresa no tuvo influencia en el diseño, recolección de los análisis, interpretación de los datos, ni en la preparación, revisión y aprobación del manuscrito).

Unidad de Endocrinología, Instituto de Nutrición y Tecnología de los Alimentos, INTA, Universidad de Chile. Unidad de Nutrición, Hospital de Calama. Departamento de Nutrición, Universidad de la Frontera, Temuco.

aNutricionista,

becnólogo Médico, MSc

Correspondencia a: Dr. Santiago Muzzo B. INTA - Universidad de Chile. Casilla 138-11. Fax: 2214030. Macul 5540 -

Macul, Santiago. E mail: smuzzo@inta.cl 
$\mathrm{E}^{1}$ bocio endémico constituye la enfermedad crónica de más fácil prevención y control. Sin embargo, aún constituye un serio problema de salud pública mundial, estimándose que alrededor de 650 millones de personas tienen bocio, 43 millones padecen de cretinismo endémico y 1.570 millones están en riesgo de padecer esta enfermedad por vivir en áreas que presentan un déficit de yodo ${ }^{1}$.

Chile tomó la decisión de erradicar el bocio endémico al promulgar, en 1959, una ley que indicó que toda sal de consumo humano debía estar yodada con una concentración de una parte de yodato de sodio o potasio por cada 10.000 partes de sal. Esta medida se implementó en 1979, fecha en la que el Ministerio de Salud dictó un decreto de obligatoriedad de yodación de la sal a todos los salineros.

Antes de iniciar la yodación de la sal en Chile, sólo existían publicaciones parcelares sobre la prevalencia de bocio en el país, las que indicaban que existía un problema moderado de bocio endémico, sin describir casos de cretinismo endémico. En 1955, se detectó $11 \%$ de bocio en escolares de Santiago ${ }^{2}$ y $19 \%$ en Coquimbo; en 1958, 33\% en Linares y $24 \%$ en Nuble 3 ; $61 \%$ y $31 \%$ en grupos cerrados de Pedregoso y Lonquimay, respectivamente; ${ }^{4}$ y en 1974, Domínguez y cols encontraron $25 \%$ en la zona de Puente Alto, Pirque y San José de Maipo ${ }^{5}$.

En 1982, Muzzo y cols detectaron una prevalencia de bocio de $18,8 \%$ en una muestra representativa de escolares de educación básica de la Región Metropolitana de Chile, la que reúne alrededor del $45 \%$ de los educandos de Chile $^{6}$. Esta prevalencia fue mayor en escolares de sexo femenino, aumentaba en ambos sexos con la edad del escolar, era mayor a menor calidad del estado nutricional, medido según la adecuación del peso para la talla, mayor a menor adecuación de la estatura del escolar y mayor en las zonas rurales y cordilleranas de la región metropolitana.

Por haberse detectado que el bocio endémico presentaba una distribución geográfica caracterizada por ser mayor en áreas rurales y cordilleranas, y para dar mayor representatividad a los estudios de escolares se decidió tomar en adelante un área de la zona norte (Calama), en centro (Santiago), en sur (Temuco) y en extremo sur (Punta Arenas) del país.
En 1986, se detectó una prevalencia de bocio de 7,6\% en escolares de educación básica de 2 zonas del país (Santiago y Temuco) ${ }^{7-9}$. Durante 1991 se repitió el diagnóstico en escolares de 3 zonas: Santiago, Temuco y Calama, detectándose una prevalencia de $11 \%$, discretamente mayor en Santiago y Temuco que en Calama. En Calama se encontró que $12 \%$ de la sal de consumo humana expendida en locales comerciales tenía una concentración menor de $60 \mathrm{ppm}$ de yodo, $27 \%$ en Santiago y $87 \%$ en Temuco. Las determinaciones de yodo en orina de estos escolares mostraron que en promedio fue $876 \mu \mathrm{g} \mathrm{I} / \mathrm{g}$ de creatinina en Calama, 752 en Santiago y 505 en Temuco. A pesar que el promedio de estas yodurias estaban sobre la recomendación vigente en ese momento, existía un porcentaje que se encontraba bajo 100 $\mu \mathrm{gI} / \mathrm{g}$ de creatinina, el que fue mayor $(10,6 \%)$ en la ciudad de Temuco ${ }^{10}$.

Desde 1993, el Instituto de Nutrición y Tecnología de los Alimentos de la Universidad de Chile certifica la adecuación de la yodación de la sal de la Salinera Punta de Lobos, la que produce cerca de $90 \%$ de la sal de consumo humano en Chile. Al inicio de este control se encontró que de las 5 plantas que tenía esta empresa, sólo la de Talcahuano tenía disminuida la concentración de yodo en la sal, planta que distribuye habitualmente su producción especialmente en la zona, llegando su distribución hasta Temuco. Esta deficiencia se corrigió en los primeros 2 meses de iniciada esta certificación.

En 1995 efectuamos un nuevo estudio de la nutrición de yodo en escolares de las 4 zonas anteriormente encuestadas, detectando que la prevalencia de bocio estaba en $9,4 \%$, la yodación de la sal en $90 \mathrm{ppm}$, aceptable para la legislación vigente. Sin embargo, las yodurias de los escolares se encontraron altas, en promedio 747 y $790 \mu \mathrm{g} \mathrm{I}$ $\mathrm{g}$ creatinina para varones y niñas respectivamente (1.849 y 1.746 en Calama; 680 y 732 en Santiago; 638 y 427 en Temuco; 563 y $545 \mu \mathrm{gI} / \mathrm{g}$ creatinina en Punta Arenas, en varones y niñas respectivamente) ${ }^{11}$.

Se entregó esta información al Ministerio de Salud, con el que se estudió la disminución de la exigencia de la yodación de la sal de consumo humano de ese momento de $100 \mathrm{ppm}$ a un promedio de $40 \mathrm{ppm}$, con un rango de 20 a 60 ppm, la cual apareció en el nuevo Reglamento 
Sanitario de los Alimentos (RSA) en enero de 2000.

Nos interesó conocer el estado actual de la nutrición de yodo del escolar chileno de educación básica después del cambio del RSA, para analizar si se cumplía el nuevo Reglamento y si se produjo el esperado descenso de las yodurias de los escolares.

\section{PACIENTES Y MÉTODO}

Durante 2001 se estudiaron 3.712 escolares chilenos en las 4 zonas estudiadas anteriormente; 833 escolares de educación básica de Calama (norte), 824 de Temuco (sur), 787 de Punta Arenas (extremo sur) y 1.268 en Santiago (centro) los que se tomaron en 2 comunas: San Bernardo y Renca ( $50 \%$ en cada una). Todos los escolares pertenecían a los establecimientos educacionales municipalizados encuestados anteriormente. Se seleccionaron aleatoriamente igual número de varones y mujeres, e igual número de alumnos por cada nivel.

Un investigador entrenado que ya había determinado la prevalencia de bocio en Chile y que se había entrenado previamente con otros investigadores de Chile y de otros países latinoamericanos, determinó por palpación la presencia, grado, consistencia y tipo de bocio de acuerdo a la clasificación de la OMS (clasificación de Pérez modificada). Se consideró bocio grado Ia cuando el tamaño de los lóbulos tiroideos era mayor que los pulgares del examinado sin visualizar el tiroides al deglutir con el cuello en posición de extensión; grado Ib cuando se palpa aumentado y se ve al deglutir en posición de extensión; grado II se palpa aumentado y se ve en posición normal del cuello y grado III cuando se ve de lejos.

A 12,5\% de los escolares encuestados se les determinó la concentración de yodo en una muestra de orina. Se tomó este porcentaje de muestras a los escolares encuestados para tener un valor de confianza sobre $95 \%$ y un error muestral menor a 5\%. La concentración de yodo en orina se determinó de acuerdo a la técnica de Wawschinek y Eber ${ }^{12}$, modificada por Pineda y cols $^{13}$. El yodo urinario se midió por espectrofotometría utilizando la reacción de Sandell Kolthoff cuyo principio se basa en la reducción del yoduro catalizada por ion cerio IV (amarillo) a Ce III (incoloro) por acción de iones de arsénico III. El cambio de color que ocurre se compara con una curva de estándares de concentración conocida. Previo a la determinación, las muestras son sometidas a una hidrólisis con ácido clórico para destruir la materia orgánica de la orina que puede interferir con la reacción ${ }^{14}$.

Los resultados se expresaron en $\mu \mathrm{g} \mathrm{I} / \mathrm{dL}$ y $\mu \mathrm{g} \mathrm{I} /$ g creatinina y se analizó su distribución como insuficiente $(<10)$, adecuada (10 a 30$)$, o excesiva en diferentes niveles de corte (30 a 60; 60-100, $>100 \mu \mathrm{gI} / \mathrm{dL}$ de creatinina).

La concentración de yodo se determinó en 304 muestras de sal, alrededor de 75 por cada localidad encuestada. Estas muestras se obtuvieron aleatoriamente en colegios, en el comercio, en hospitales y en las casas particulares de los escolares encuestados. La concentración de yodo en la sal se cuantificó por la técnica de Pineda y cols ${ }^{15}$. Los resultados se expresaron en $\mu \mathrm{g} \mathrm{I} / \mathrm{g}$ sal, como promedios $\mathrm{y}$ porcentajes de muestras bajo y sobre el rango de exigencia actual del RSA (20 a $60 \mu \mathrm{g} \mathrm{I} / \mathrm{g} \mathrm{sal})$.

Estadística. El análisis de los resultados se efectuó por la prueba de chi cuadrado y por el test no paramétrico de Kruskal-Wallis.

\section{RESULTADOS}

La Tabla 1 muestra que la prevalencia de bocio en la muestra total fue de $6,4 \%$, con una tendencia no significativa a ser discretamente mayor en las mujeres $(6,8 \%)$ que en los varones (5,9\%).

Tabla 1. Prevalencia de bocio en cuatro zonas censorias de Chile en el año 2001

\begin{tabular}{|lcccccc|}
\hline & \multicolumn{2}{c}{ Sin bocio } & \multicolumn{2}{c|}{ Con bocio } & \multicolumn{2}{c|}{ Totales } \\
& $\mathrm{n}$ & $\%$ & $\mathrm{n}$ & $\%$ & $\mathrm{n}$ & $\%$ \\
\hline Varones & 1.738 & 94,0 & 110 & 5,9 & 1.848 & 100,0 \\
Mujeres & 1.736 & 93,1 & 128 & 6,8 & 1.864 & 100,0 \\
Totales & 3.474 & 93,6 & 238 & 6,4 & 3.712 & 100,0 \\
\hline
\end{tabular}

$\chi^{2}=1,29 \quad 1 \mathrm{gl} \quad \mathrm{p}=\mathrm{NS}$ 
La Tabla 2 muestra la prevalencia de bocio según el área geográfica estudiada y el grado del bocio. Se detectó 7,3\% de bocio en Calama, 5,9\% en Santiago, 5,9\% en Temuco y 6,7\% en Punta Arenas, sin detectar diferencias significativas entre estos valores. Se aprecia que la mayoría de los bocios fue grado Ia $(5,0 \%)$, siendo grado Ib sólo $1,2 \%$, grado II $0,2 \%$ y grado III sólo $0,1 \%$. Se encontró sólo un bocio grado III en un escolar de sexo femenino de Punta Arenas y otro en Calama.

La Tabla 3, indica la concentración de yodo en las muestras de sal recolectadas. Se aprecia que los promedios son bastante adecuados para la reglamentación vigente en las 4 zonas estudiadas, siendo algo menor en Calama $(25,4 \mu \mathrm{g} \mathrm{I} / \mathrm{g} \mathrm{sal})$, que en Santiago, Temuco y Punta Arenas (36,2; 27,7 y $33,4 \mu \mathrm{g} \mathrm{I} / \mathrm{g}$ sal respectivamente). En Calama $28 \%$ de las muestras estaba bajo y $17 \%$ sobre el rango de aceptación ( 20 a $60 \mu \mathrm{g} \mathrm{I} / \mathrm{g} \mathrm{sal}$ ), mientras que en Santiago, Temuco y Punta Arenas estaban bajo $6,2 \%$; 26,6 y $16,2 \%$ respectivamente y sobre el rango $0 \%, 1,6 \%$ y $3,2 \%$ respectivamente.

La Tabla 4, presenta las excreciones urinarias de yodo según localidad y sexo, expresado en $\mu \mathrm{g}$ $\mathrm{I} / \mathrm{dL}$ y $\mu \mathrm{g} \mathrm{I/g}$ creatinina. Se aprecia que esta excreción fue muy alta en Calama, tanto en varones y mujeres (2.838 y $2.852 \mu \mathrm{g} \mathrm{I} / \mathrm{g}$ creatinina, respectivamente) y significativamente mayor que en las otras áreas, las que también estaban elevadas en ambos sexos, de acuerdo a la recomendación de $150 \mu \mathrm{g}$ de ingesta de yodo por día; (700 y 837 en Santiago; 1.318 y 1.438 en Temuco y 407 y 312 en Punta Arenas, respectivamente).

La Tabla 5, indica que $89,6 \%$ de las yodurias de los varones y $79,7 \%$ de las mujeres tenían una concentración de yodo superior a $30 \mu \mathrm{g} / \mathrm{dL}$, y $0,5 \%$ de los varones y ninguna niña presentó una yoduria bajo $10 \mu \mathrm{g} / \mathrm{dL}$. Se encontraron diferencias significativas ( $p<0,01)$ entre varones y mujeres.

Tabla 2. Prevalencia de bocio en Chile en el año 2001, según localidad

\begin{tabular}{|c|c|c|c|c|c|c|c|c|c|c|}
\hline \multirow{2}{*}{$\begin{array}{l}\text { Grado } \\
\text { bocio }\end{array}$} & \multicolumn{2}{|c|}{ Calama } & \multicolumn{2}{|c|}{ Santiago } & \multicolumn{2}{|c|}{ Temuco } & \multicolumn{2}{|c|}{ Punta Arenas } & \multicolumn{2}{|c|}{ Totales } \\
\hline & $\mathrm{n}$ & $\%$ & $\mathrm{n}$ & $\%$ & $\mathrm{n}$ & $\%$ & $\mathrm{n}$ & $\%$ & $\mathrm{n}$ & $\%$ \\
\hline 0 & 772 & 92,7 & 1.193 & 94,1 & 775 & 94,1 & 734 & 93,3 & 3.474 & 93,5 \\
\hline Ia & 43 & 5,2 & 64 & 5,0 & 39 & 4,7 & 38 & 4,8 & 184 & 5,0 \\
\hline $\mathrm{Ib}$ & 16 & 1,9 & 10 & 0,8 & 8 & 1,0 & 12 & 1,5 & 46 & 1,2 \\
\hline II & 1 & 0,1 & 1 & 0,1 & 2 & 0,2 & 2 & 0,3 & 6 & 0,2 \\
\hline III & 1 & 0,1 & 0 & 0,0 & 0 & 0,0 & 1 & 0,1 & 2 & 0,1 \\
\hline \multicolumn{11}{|l|}{ Total con } \\
\hline bocio & 61 & 7,3 & 75 & 5,9 & 49 & 5,9 & 53 & 6,7 & 238 & 6,5 \\
\hline Totales & 833 & 100,0 & 1.268 & 100,0 & 824 & 100,0 & 787 & 100,0 & 3.712 & 100,0 \\
\hline
\end{tabular}

$\chi^{2}=2,11 \quad 3 \mathrm{gl} \quad \mathrm{p}=\mathrm{NS}$

Tabla 3. C oncentracion de yodo en la sal en el año 2001, según localidad

\begin{tabular}{|c|c|c|c|c|c|}
\hline & \multirow{3}{*}{$\mu \mathrm{g} \mathrm{I} / \mathrm{g}$ sal } & \multicolumn{4}{|c|}{ Muestras } \\
\hline & & \multicolumn{2}{|c|}{$<20 \mu \mathrm{g} \mathrm{I} / \mathrm{g} \mathrm{sal}$} & \multicolumn{2}{|c|}{$>60 \mu \mathrm{g} \mathrm{I} / \mathrm{g} \mathrm{sal}$} \\
\hline & & $\mathrm{n}$ & $\%$ & $\mathrm{n}$ & $\%$ \\
\hline Calama & $25,4 \pm 10,0(57)^{*}$ & 16 & 28,0 & 1 & 1,7 \\
\hline Santiago & $36,2 \pm 10,6(64)$ & 4 & 6,2 & 0 & 0,0 \\
\hline Temuco & $27,7 \pm 11,6(60)$ & 16 & 26,6 & 1 & 1,6 \\
\hline Punta Arenas & $33,4 \pm 13,6(123)$ & 20 & 16,2 & 4 & 3,2 \\
\hline
\end{tabular}

*promedio $\pm \mathrm{DE}$ (número de muestras). 


\section{DisCUSIÓN}

La prevalencia actual de bocio en escolares de educación básica en 4 zonas de Chile, fue 6,4\%, situación que se ha mantenido a nivel similar a la detectada en la última investigación realizada en 19947,10, existiendo una tendencia no significativa a una menor prevalencia en los varones que en las mujeres (5,9 y 6,8\% respectivamente).

La mantención de una baja prevalencia de bocio (levemente superior a 5\%) en los escolares de estas zonas durante los últimos 10 años, sumado a que las últimas investigaciones demuestran una ingestión de yodo sobre lo recomendado para este grupo etáreo (150 $\mu$ g de yodo por día), indicaría que ya se ha solucionado este problema nutricional colectivo ${ }^{16}$.

Las sales recolectadas en las zonas presentaron una concentración aceptable de yodo, de acuerdo a la exigencia actual del RSA; $18 \%$ de las muestras estuvieron bajo el rango y sólo $2 \%$ sobre el rango de aceptación.

El porcentaje de bocio detectado por palpación en los escolares de este estudio fue discretamente superior a $5 \%$, cifra sobre la cual la OMS cataloga el problema como endémico en escolares de educación básica. En otro estudio de prevalencia en 10 ciudades de Chile el año 1999 efectuado por el proyecto Thyromobil, se detectó mediante un ecógrafo de alta sensibilidad una prevalencia de bocio de 5,3\%, mientras que por palpación del tiroides se detectó en estos mismos escolares una prevalencia de bocio similar a los estudios anteriormente señalados.
Esto podría explicarse por la dificultad de diferenciar a la palpación un tiroides normal de uno con bocio grado Ia. Esta situación se da especialmente en los púberes, período en el que normalmente hay un mayor crecimiento tiroideo además la mayoría de los bocios detectados en este estudio fueron de este grado. Sin embargo,

\section{Tabla 4. Excreción de yodo en orina,} según localidad

\begin{tabular}{|lcc|}
\hline & $\mu \mathrm{g} / \mathrm{l}$ & $\mu \mathrm{g} \mathrm{I} / \mathrm{g}$ creatinina \\
\hline Calama & $2.579 \pm 802$ & $2.666 \pm 2.063$ \\
& $(102)$ & $(102)$ \\
Santiago & $738 \pm 415$ & $729 \pm 518$ \\
& $(162)$ & $(162)$ \\
Temuco & $981 \pm 393$ & $1.282 \pm 669$ \\
& $(71)$ & $(71)$ \\
Punta Arenas & $335 \pm 160$ & $384 \pm 355$ \\
& $(109)$ & $(99)$ \\
Totales & $1.096 \pm 400$ & $1.191 \pm 685$ \\
& $(447)$ & $(437)$ \\
\hline
\end{tabular}

Test de Kruskal-Wallis: (a) yoduria en $\mu \mathrm{g} \mathrm{I} / \mathrm{dl}$ por zona. $\chi^{2}=313 p<0,0001$. Temuco vs Santiago $p<0,00$; Calama vs Punta Arenas $p<0,00$; Temuco vs Calama $p$ $<0,00$; Temuco vs Punta Arenas $p<0,00$; Santiago vs Punta Arenas $p<0,00$; Santiago vs Calama $p<0,00$. b) yoduria en $\mu \mathrm{g} \mathrm{I} / \mathrm{g}$ creatinina por zona. $\chi^{2}=255.95 \mathrm{p}$ $<0,0001$. Calama vs Punta Arenas $p<0,00$; Temuco vs Santiago $p<0,00$; Temuco vs Calama $p<0,00$; Temuco vs Punta Arenas $p<0,00$; Santiago vs Calama $p<0,00$; Temuco vs Santiago $\mathrm{p}<0,00$.

Tabla 5. D istribución de las yodurias en el año 2001, según concentración y sexo

\begin{tabular}{|lrrrrrr|}
\hline \multirow{2}{*}{$\mu \mathrm{g} \mathrm{I} / \mathrm{dl})$} & \multicolumn{2}{c}{ Varones } & \multicolumn{2}{c}{ Mujeres } & \multicolumn{2}{c|}{ dTotal } \\
& $\mathrm{n}$ & $\%$ & $\mathrm{n}$ & $\%$ & $\mathrm{n}$ & $\%$ \\
\hline$<10$ & 1 & 0,5 & 0 & 0,0 & 1 & 0,2 \\
$10-29$ & 21 & 99 & 51 & 20,3 & 72 & 15,5 \\
$30-59$ & 50 & 23,5 & 59 & 23,5 & 109 & 23,5 \\
$60-99$ & 56 & 26,3 & 42 & 16,8 & 98 & 21,1 \\
$\geq 100$ & 85 & 39,8 & 99 & 39,4 & 184 & 39,8 \\
Totales & 213 & 100,0 & 251 & 100,0 & 464 & 100,0 \\
\hline
\end{tabular}

$\chi^{2}=14,29 \quad 4 \mathrm{gl} \quad \mathrm{p}<0,01$ 
habría que estar atentos a la posibilidad de que aumente la prevalencia de bocio por la ingesta excesiva de yodo que ha habido en nuestro país, ya que se ha descrito en poblaciones que ingieren un exceso de yodo un aumento de la prevalencia de bocio por tiroiditis autoinmune ${ }^{16}$.

Las enfermedades tiroideas autoinmunes son enfermedades poligénicas, en las cuales la penetrancia está influenciada fuertemente por factores ambientales. Tanto el déficit como el exceso de yodo pueden conducir a una enfermedad autoinmune tiroidea ${ }^{17}$.

Pocas poblaciones tienen alto consumo de yodo de fuentes naturales; esta situación se produjo en Japón por alto consumo de algas ${ }^{18}$, en China por consumo de agua con alto contenido de yodo ${ }^{19}$, en Islandia por el uso de pescado para la alimentación de ganado que elevó el contenido de yodo en la carne y derivados lácteos ${ }^{20}$.

Otra causa es una suplementación de yodo descontrolada y por químicos, que contienen yodo, usados para la preparación de comidas (acondicionadores de los panaderos) y algunos medicamentos ${ }^{21,22}$.

En la costa de Hokkaido, en Japón, que tienen una dieta rica en algas, las yodurias de pacientes con bocio eran muy elevadas. En algunas áreas, $14 \%$ tenía bocio, el que disminuyó al bajar la ingesta de yodo ${ }^{18}$. En China se compararon niños de 2 poblaciones; una con una ingesta de yodo en el agua potable de 54 con otra de $462 \mu \mathrm{g} / \mathrm{L}$, las yodurias eran de 428 y $1.235 \mu \mathrm{g} / \mathrm{g}$ creatinina respectivamente. El área con la mayor ingesta era una área con bocio endémico, donde la TSH se encontró elevada (en promedio 7,8 mU/L) ${ }^{19}$.

$\mathrm{Al}$ introducir en los Estados Unidos de América, yodato en la industria del pan, por un período se produjo una ingesta alta de yodo en la población que varió de 500 a $1.000 \mu \mathrm{g} /$ día. Durante la ingestión baja de yodo tenían una incidencia alta de hipertiroidismo no autoinmune aun hasta 20 años después de elevar la ingesta de yodo. Los niveles altos de TSH se daban más con ingesta alta de yodo ${ }^{23}$.

En modelos animales se ha demostrado que el paso de una baja a una alta ingesta de yodo produce tiroiditis ${ }^{24}$. En humanos, se ha demostrado en biopsias de tiroides, un aumento histológico de tiroiditis al subir el consumo de yodo 25 .
En una población japonesa normal se demostró que no más de $2 \%$ tenían anticuerpos antitiroideos positivos ${ }^{18}$. En Grecia se detectó que en áreas con excreción urinaria de yodo de $200 \mu \mathrm{g} / \mathrm{L}$, $16 \%$ de los pacientes con bocio tenían anticuerpos antitiroideos positivos, lo que era negativo en pacientes con bocio de áreas con baja ingesta (74 $\mu \mathrm{g} / \mathrm{L})^{26}$. Otro grupo griego encontró que adultos con bocio no tóxico, que recibieron $480 \mathrm{mg}$ de aceite yodado intramuscular, sus yodurias subieron de 142 a $859 \mu \mathrm{g} / \mathrm{g}$ creatinina a los 3 meses para luego descender, pero con un aumento significativo de los anticuerpos antitiroideos, concluyendo que el exceso de yodo induce o por lo menos facilita la expresión de una enfermedad autoinmune en individuos susceptibles ${ }^{27}$.

A pesar de todas estas evidencias que indican que la ingestión elevada y mantenida de yodo facilitaría la expresión de problemas tiroideos autoinmunes, otros autores no han podido certificar esta correlación. Knobel y cols, en 1986, no encontraron aumento de anticuerpos antitiroideos en personas de una zona de bocio endémico tratada con aceite yodado ${ }^{28}$. En 1998, Michaud y Téllez no encontraron aumento de bocio en escolares de Pirque en Chile, ni alteración de su función tiroidea a pesar de presentar una excreción promedio de yodo de $1.580 \mu \mathrm{g} / \mathrm{L}^{29}$.

La prevalencia actual de bocio en los escolares de este estudio fue similar en las diferentes zonas , analizadas. La mayor prevalencia de bocio encontrada de norte a sur del país y por sexo en encuestas anteriores, también se han perdido posiblemente por la baja prevalencia actual de bocio 7,10

Es interesante destacar que la excreción urinaria de yodo medida en los escolares de este estudio fue muy alta, siendo tres veces mayor en Calama que en las otras localidades. No contamos con una explicación para este hallazgo, pero pensamos que es importante descartar una fuente de contaminación ambiental. Nuestras investigaciones previas indican que las yodurias en los escolares de Calama subieron de $720 \mu \mathrm{g} \mathrm{I} / \mathrm{L}$ en 1986 a $1.690 \mu \mathrm{g} \mathrm{I} / \mathrm{L}$ en 1994. Si bien, no detectamos un mayor porcentaje de bocio en los escolares de Calama con yodurias sobre $1.000 \mu \mathrm{g} \mathrm{I} / \mathrm{g}$ creatinina, habría que estar vigilante de la nutrición de yodo de estos escolares, ya que desconocemos el tiempo que se requiere para que una 
ingesta alta de yodo desencadene un aumento del porcentaje de bocio.

Con los resultados obtenidos en este estudio se concluye que el bocio endémico ha dejado de ser un problema nutricional colectivo en Chile. Esto se expresaría en la baja prevalencia de bocio detectada en los escolares de educación básica en los últimos 10 años en nuestro país y en la adecuada yodación de la sal de consumo humano de acuerdo a la exigencia del Reglamento Sanitario de los Alimentos vigente. Sin embargo, aún no tiene explicación el aumento de la excreción de yodo en orina pesquisado en estos escolares, al compararlo con el estudio anterior efectuado en las mismas 4 zonas en 1995 y a

\section{REFERENCIAS}

1. ICCIDD/UNICEF/WHO. Global prevalence of iodine deficiency disorders. MDIS Working paper \#1. Micronutrient Information System, Ginebra, Organización Mundial de la Salud, 1993.

2. Donoso F, Jadresic A, López E, García de los Ríos M, Concha J, Espejo M et al. Encuesta de bocio en escolares de la provincia de Santiago y consideraciones sobre el problema de bocio endémico. Rev Méd Chile 1955; 83 (Suppl).

3. Donoso F, Lennon H, Canessa M, Valuejos J, Aldunate G. Encuesta de bocio en escolares de la provincia de Linares y Ñuble. Rev Méd Chile 1959; 87: 717.

4. Nagel R, Etcheverri R, Guzmán C, Hime A, Barzelatto J, Covarrubias E. Encuesta de bocio endémico en la población mapuche y en algunos colegios de primera enseñanza de las provincias de Cautín y Malleco. Rev Méd Chile 1962; 90: 6168.

5. Dominguez M, Quesney F, Michaud P, Maggiolo G, UGARTE JM. La prevalencia del bocio en escolares de las comunas de Puente Alto, Pirque y San José de Maipo: estudio clínico epidemiológico. Rev Méd Chile 1974; 102: 633.

6. Muzzo S, Leiva L, Zvaighaft A. Características del bocio endémico en el escolar de la Región Metropolitana de Chile. Rev Chil Nutr 1985; 13: 143-7. pesar que el RSA bajó la exigencia de la concentración de yodo en la sal de consumo humano de 100 a $40 \mathrm{ppm}$. La variación de la excreción urinaria de yodo en escolares en las diferentes zonas, sugiere una posible contaminación ambiental (alimentos, agua o sustancias inhaladas) hecho necesario de investigar. Se considera de mucha importancia mantener una vigilancia continua de la nutrición de yodo en nuestro país, con la finalidad de descartar problemas tiroideos por ingesta excesiva de yodo y además se recomienda estudiar la concentración de yodo en diferentes alimentos para buscar una explicación a la falta de descenso de las yodurias de los escolares en Calama, Santiago y Temuco.

7. Muzzo S, Pozo M, Zvaighaft A, Rodewald AM, BIOLEy E, Leiva L Características actuales del bocio endémico en escolares de dos zonas censoras de Chile. Rev Chil Nutr 1989; 17: 60-5.

8. Leiva L, Pozo M, Biowey E, Muzzo S. Excreción de yodo urinario y función tiroidea de escolares de Santiago y Temuco. Rev Chil Nutr 1992; 20: 38-42.

9. Pozo M, Rodewald AM, Biolley E, Zvaighaft A, LeIva L, Muzzo S. Prevalencia de bocio endémico en escolares del centro y sur de Chile. Rev Chil Ped 1989; 60: 359-62.

10. Muzzo S, Burgueño M, Carvajal F, Moreno R, Leiva $\mathrm{L}$. Características actuales de bocio endémico en 3 zonas censorias de Chile. Arch Latinoam Nutr 1994; 44: 82-6.

11. Muzzo S, Burgueño M, Carvajal F, Bioley E, Avendaño M, Vargas A et al. Nutrición de yodo en escolares de 4 zonas censoras de Chile. Rev Méd Chile 1997; 125: 1299-304.

12. Wawschinek O, Eber O, Petek P, Wakoning $P$, GURAKER A. Bestimmung der Harnjodausseheidung mittels einer modifizierten Cer-Arsenit methode. Berichte der OGKC 1985; 8: 13-5.

13. Pineda O, Morales S, Sosa J. Determinación de yodo en orina. Sección de Bioquímica Nutricional. Instituto de Nutrición de Centroamérica y Panamá (INCAP). Documento Interno, 1989.

14. Dunn J, Crutchiteld H, Gutenkunst R, Dunn A. Methods for measuring iodine in urine. International Council for Control of Iodine Deficiency 
Disorders. 1993. ICCIDD/UNICEF/WHO Publicación ISBN 90-70785-12-9.

15. Pineda O, Dary O, Morales S. Método de campo para la determinación de yodato de sal. Sección de bioquímica nutricional. Instituto de Nutrición de Centroamérica y Panamá (INCAP). Documento Interno, 1981.

16. Bailey KV, Clugston GA. Iodine deficiency disorders, En: Murray CJL, López AD, eds. The global burden of disease and risk factors in 1990. OMS/ Banco Mundial, Ginebra, Organización Mundial de la Salud (por publicar).

17. Ruwhof C, Drexhage HA. Iodine and thyroid autoimmune disease in animal models. Thyroid 2001; 11: 427-36.

18. Inoue M, Taketani N, Sato T, Nakajima H. High incidence of chronic lymphocytic thyroiditis in apparently healthy school children: Epidemiological and clinical study. Endocrinol Jpn 1975; 22: 483-8.

19. Wong GWK, Lam CWK, Kwok MY, MaK TW, Aнuja AT, CHOw CC ET AL. Childhood goiter and urinary iodine excretion in Hong Kong. Eur J Pediatr 1998; 157: 8-12.

20. Lauberg P, Pedersen KM, Vestergaard H, Sigurdsoson G. High incidence of multinodular toxic goiter in elderly population in low iodine intake area vs high incidence of Grave's disease in the young in high iodine intake area: comparative surveys of thyrotoxicosis epidemilogy in East-Jutland Denmark and Iceland. Intern Med 1991; 229: 415-20.

21. WeEtman AP. Autoimmune thyroiditis: predisposition and pathogenesis. Clin Endocrinol (Oxf) 1992; 36: 307-23.
22. Ishizukiy $A$, Hirooka $Y$, Tanigawa $S$, Sawai $Y$. The prevalence of antithyroid antibodies and long term follow up in patients with lipiodol iodine induced goiter. Nipppon Naibunpi Gakkai Zasshi 1994; 70: 957-66.

23. Vagenakis AG, Wang CA, Burger A, Maloof F, Braverman LE, IngBar SH. Iodine induced thyrotoxicosis in Boston. N Engl J Med 1972; 287: 523-7.

24. Sundic R, Bagchi N, Brown TR. The role of iodine in thyroid autoimmunity from chickens to humans: a review. Autoimmunity 1992; 13: 61-8.

25. Harach HR, Escalante DA, Onativia A, Outes JL, DAY ES, WILAMS ED. Thyroid carcinoma and thyroiditis in endemic goiter region before and after iodine prophylaxis. Acta Endocrinol (Copenh) 1985; 108: 55-60.

26. Tsatsouls A, Johnson EO, Andricula M, Kalogera C, Svarna E, Spyroy P et al. Thyroid autoimmunity is associated with higher urinary iodine concentrations in an iodine deficient area of Northwestern Greece. Thyroid 1999; 9: 279-83.

27. Papanastasiou L, Alevizaki M, Peperingos G, Mantzos E, Tseient-Balafouta S, Koutras DA. The effect of iodine administration on the development of thyroid autoimmunity in patients with nontoxic goiter. Thyroid 2000; 10: 493-8.

28. KNoBel M, Medeiros-Neto G. Iodized oil treatment for endemic goiter does not induce the surge of positive serum concentrations of antithyroglobulin or antimicrosomal autoantibodies. J Endocrinol Invest 1986; 9: 321-4.

29. Michaud P, Telez R. Thyroid function in a population with an extra iodine intake. Rev Méd Chile 1998; 126: 177-82.

Agradecimientos

Se agradece el apoyo de la empresa Salinas Punta de Lobos para la realización de este Proyecto. 